Article

\title{
Strongly Focused Circularly Polarized Optical Vortices Regulated by a Fractal Conical Lens
}

\author{
Zhirong Liu ${ }^{1,2, *,+} \mathbb{D}$, Kelin Huang ${ }^{1,+}$, Anlian Yang ${ }^{1}$, Xun Wang ${ }^{1, *}$ and Philip H. Jones ${ }^{2} \mathbb{C}$ \\ 1 Department of Applied Physics, East China Jiaotong University, Nanchang 330013, China; \\ huangklecjtu@126.com (K.H.); yangalecjtu@126.com (A.Y.) \\ 2 Department of Physics and Astronomy, University College London, Gower Street, London WC1E 6BT, UK; \\ philip.jones@ucl.ac.uk \\ * Correspondence: liuzhirong_2003@126.com (Z.L.); wangxecjtu@126.com (X.W.) \\ + These authors contributed equally to this work.
}

Received: 30 October 2019; Accepted: 16 December 2019; Published: 18 December 2019

check for updates

\begin{abstract}
In this paper, a recently-proposed pure-phase optical element, the fractal conical lens (FCL), is introduced for the regulation of strongly-focused circularly-polarized optical vortices in a high numerical aperture (NA) optical system. Strong focusing characteristics of circularly polarized optical vortices through a high NA system in cases with and without a FCL are investigated comparatively. Moreover, the conversion between spin angular momentum (SAM) and orbital angular momentum (OAM) of the focused optical vortex in the focal vicinity is also analyzed. Results revealed that a FCL of different stage $S$ could significantly regulate the distributions of tight focusing intensity and angular momentum of the circularly polarized optical vortex. The interesting results obtained here may be advantageous when using a FCL to shape vortex beams or utilizing circularly polarized vortex beams to exploit new-type optical tweezers.
\end{abstract}

Keywords: laser beam shaping; polarization; image formation theory

\section{Introduction}

In recent years, a rapid growing research interest has been attracted in the tight focusing of various polarized light waves, optical vortices, and laser pulses through a high numerical aperture (NA) objective lens, because of its unique properties and potential applications in single molecule imaging, microscopy, and optical manipulation [1-17]. On the other hand, as circularly polarized optical vortices could be decomposed into radial and azimuthal polarization, then with proper combination of vortex charge and circular polarization handedness, a focusing field with extremely strong longitudinal component and flat-topped profile could be generated [5].

Over the past several decades, angular momentum (AM) has been regarded as one of the important properties of the light field since the groundbreaking work performed by Poynting and Allen [18,19]. In the years that followed, researchers realized that photons may carry two different kinds of angular moments, i.e., spin angular momentum (SAM) and orbital angular momentum (OAM). Not long ago, it was reported that SAM may transform into OAM in inhomogeneous anisotropic media [20]. Furthermore, it was found that the conversion of spin-to-orbital angular momentum could also take place in a homogeneous medium by means of a high NA objective lens [21].

Fractal conical lens (FCL) is a recently-proposed pure-phase optical element, of which radial phase profile is designed following a certain kind of Cantor set (CS) [22,23]. Owing to its distinguishing properties that multiple foci could be produced along the optical axis under monochromatic illumination, the FCL has found applications in several branches of modern optics, such as laser beam shaping, offering optical guiding, and exploiting new type optical tweezers [22-24]. In this work, we would introduce 
the FCL to regulate the tightly-focused circularly-polarized optical vortices. Following the generalized vector Debye integral, the tight focusing characteristics of circularly polarized optical vortices through a high NA optical system in cases with and without a FCL are investigated comparatively. Moreover, the conversion between the SAM and OAM of focused optical vortices in the focal vicinity is also analyzed. Additionally, influence of several relevant factors, such as circular polarization direction $\sigma_{\mathrm{z}}$, vortex beam's topological charge $m$, FCL's stage $S$, and objective lens' $N A$, on the optical vortex's focusing intensity distribution and conversion between SAM and OAM in the focal vicinity are investigated in detail. Results reveal that a FCL of different stage $S$ could significantly modulate the tight focusing intensity distribution of circularly polarized vortex beams. Results obtained here may find applications when using a FCL to shape optical vortices or utilizing circularly polarized vortex beams to generate new-type optical tweezers.

\section{Theory Model}

Fractal conical lens (FCL) is known as a more general diffractive optical device than conical lens (CL), which has a rotational symmetric structure and special radial phase envelope designed following a kind of Cantor set (CS) of a certain stage $S[22,23]$. In order to visualize the structure of a FCL, Figure 1a plots the schematic of a one-dimensional (1-D) fractal structure of a FCL following the triadic $\mathrm{CS}$, and Figure $1 \mathrm{~b}$ presents the two-dimensional $(2-\mathrm{D})$ fractal structure of a FCL $(S=3)$ based on the triadic CS. Here, it is notable that a CS other than triadic could also be formed with a similar process.

(a)

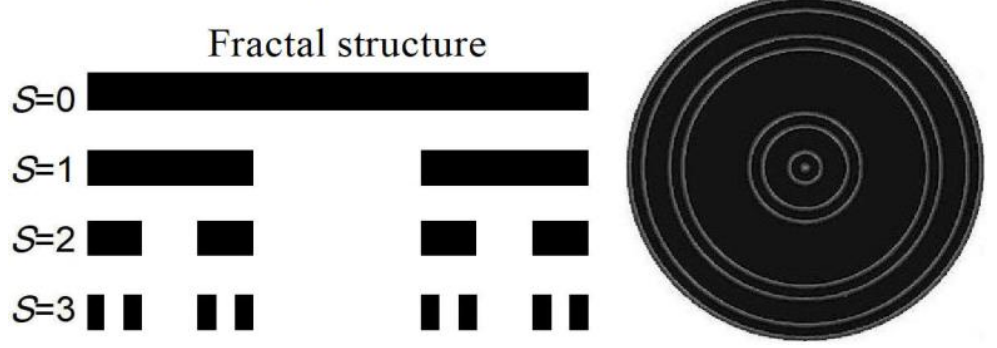

Figure 1. (a) Schematic of a 1-D fractal structure of a FCL following the triadic CS, starting from the stage $S=0$ to $S=3$; (b) schematic of 2-D fractal structure of a FCL $(S=3)$ following the triadic CS.

According to its special fractal structure, the Cantor function $F_{S}(x)$ could be defined by $[22,23]$

$$
F_{S}(x)=\left\{\begin{array}{ll}
\frac{l}{2^{S}} & \text { if } p_{S, l} \leq x \leq q_{S, l} \\
\frac{1}{2^{S}} \frac{x-q_{S, l}}{p_{S, l+1}-q_{S, l}}+\frac{l}{2^{S}} & \text { if } q_{S, l} \leq x \leq p_{S, l+1}
\end{array},\right.
$$

where $S$ and $l$, respectively, represent the Cantor function's stage and the number of disjoint gaps intervals $\left[p_{S, l}, q_{S, l}\right]$, with $S=0,1,2, \ldots$ and $l=1, \ldots, 2^{S-1} . q$ and $p$, respectively, stand for the start and end points for each subsection of Cantor function.

As illuminated by monochromic light waves, the FCL's transmittance function could be given by

$$
T(\theta)=\exp \left[-\mathrm{i} 2^{S+1} \pi F_{S}\left(\frac{\sin \theta}{\sin \alpha}\right)\right]
$$

where $\alpha=\sin ^{-1}\left(N A / n_{1}\right)$ represents the maximum value of convergence angle $\theta$ related to NA and $n_{1}$. Here, $n_{1}$ denotes the refractive index in the image space.

In this paper, tight focusing characteristics of a circularly polarized optical vortex through a high NA optical system in cases with and without a FCL are analytically examined and numerically 
illustrated. Following the generalized vector Debye integral [22], for circularly polarized optical vortex incidence, the electric field in the focal vicinity has the form [25]

$$
\begin{aligned}
E(r, \varphi, z)= & {\left[\begin{array}{c}
E_{x} \\
E_{y} \\
E_{z}
\end{array}\right]=-\frac{i k f}{2 \pi} \int_{0}^{\alpha} \int_{0}^{2 \pi} A(\theta) T(\theta) \exp (i m \phi) \sin \theta \sqrt{\cos \theta} } \\
& \times \exp [i k(z \cos \theta+r \sin \theta \cos (\phi-\varphi))] \\
& \times\left[\begin{array}{c}
\left(\cos ^{2} \phi \cos \theta+\sin ^{2} \phi\right) \pm i \cos \phi \sin \phi(\cos \theta-1) \\
\cos \phi \sin \phi(\cos \theta-1) \pm i\left(\cos ^{2} \phi+\sin ^{2} \phi \cos \theta\right) \\
\sin \theta \exp ( \pm i \phi)
\end{array}\right] \\
& \times d \phi d \theta .
\end{aligned}
$$

where $A(\theta)$ denotes the apodization function. $T(\theta)$ represents the transmittance function of a FCL. After tedious integral calculations, the $x-, y$-, and $z$-polarized components of an electric field in the focal vicinity could be obtained, respectively, as

$$
\begin{aligned}
E_{ \pm, x}(r, \varphi, z)= & -\frac{i k f}{2} \int_{0}^{\alpha} A(\theta) T(\theta) \sin \theta \sqrt{\cos \theta} \exp (i k z \cos \theta) \\
& \times\left[(1+\cos \theta) i^{m} J_{m}(k r \sin \theta) \exp (i m \varphi)\right. \\
& +(\cos \theta-1) i^{m \pm 2} J_{m \pm 2}(k r \sin \theta) \\
& \times \exp [i(m \pm 2) \varphi]] d \varphi, \\
E_{ \pm, y}(r, \varphi, z)= & -\frac{i k f}{2} \int_{0}^{\alpha} A(\theta) T(\theta) \sin \theta \sqrt{\cos \theta} \exp (i k z \cos \theta) \\
& \times\left[ \pm i \times(1+\cos \theta) i^{m} J_{m}(k r \sin \theta) \exp (i m \varphi)\right. \\
& \pm i \cdot(\cos \theta-1) i^{m \pm 2} J_{m \pm 2}(k r \sin \theta) \\
& \times \exp [i(m \pm 2) \varphi]] d \varphi, \\
E_{ \pm, z}(r, \varphi, z)= & -i k f \int_{0}^{\alpha} A(\theta) T(\theta) \sin ^{2} \theta \sqrt{\cos \theta} \exp (i k z \cos \theta) \\
& \times i^{m \pm 1} J_{m \pm 1}(k r \sin \theta) \exp [i(m \pm 1) \varphi] d \varphi,
\end{aligned}
$$

where $E_{+, i}(r, \varphi, z)$ and $E_{-, i}(r, \varphi, z)(i=x, y, z)$ represent the right-hand circularly (RHC) polarized and left-hand circularly (LHC) polarized electric field, respectively. $r, \varphi$ and $z$ denote the cylindrical coordinates of an observation point. $k=2 \pi / \lambda$ stands for the wave vector, $f$ is the high NA objective lens' focal length, $\phi$ is the azimuthal angle of incident beam, and $\theta$ is the convergence angle that varies from 0 to the maximum convergence angle $\alpha . J_{m}(\cdot)$ is a first kind Bessel function of order $m$.

For simplicity, the incident light wave's mode index is assumed to be 0 , and then the transversal amplitude of the Laguerre-Gaussian beam $\mathrm{LG}_{0}^{m}$ could be given by [21]

$$
E_{m}(r)=\left(\frac{\sqrt{2} r}{w_{0}}\right)^{|m|} \exp \left(-\frac{r^{2}}{w_{0}^{2}}\right) .
$$

Obeying the sine condition, i.e., $r=f \sin \theta$, the apodization function could be written as

$$
E_{m}(\theta)=\left(\frac{\sqrt{2} f \sin \theta}{w_{0}}\right)^{|m|} \exp \left(-\frac{f^{2} \sin ^{2} \theta}{w_{0}^{2}}\right) .
$$

Equations (2), (4)-(6), and (8) provide a general description of a circularly polarized optical vortex through a high NA objective lens system with a FCL. In the following discussion, these equations are used to investigate the distributions of intensity, phase, and intensity evolution of strongly focused circularly polarized optical vortex in cases with and without a FCL. In addition, the 
spin-to-orbital angular momentum conversion for optical vortex in such a strongly focused optical system is be analyzed. In the following numerical simulations, the common parameters are chosen as: $\lambda=0.632 \mu \mathrm{m}, f=1 \mathrm{~mm}$, and $n_{1}=1.33$.

\section{Numerical Simulations and Analysis}

In terms of the analytical expression derived above, we graphically illustrate the tight focusing characteristics of circularly polarized optical vortex in cases with and without a FCL. Figure 2a-c, respectively, plot the intensity distributions of $x$-polarized component, $y$-polarized component, and $z$-polarized component $\left|\mathrm{E}_{\mathrm{i}}\right|^{2}(i=x, y, z)$ of a strongly focused RHC polarized Gaussian beam $\left(\sigma_{z}=+1\right.$, $m=0)$ without a FCL. For a RHC polarized Gaussian beam $\left(\sigma_{z}=+1, m=0\right)$, it can be seen from Figure $2 \mathrm{a}-\mathrm{c}$ that in the focal plane, both $x$-polarized component and $y$-polarized component present peak-centered intensity distributions, whereas the z-polarized component forms a donut-shaped configuration. Thus, the total intensity distribution forms an on-axis-maximum profile (Figure $2 \mathrm{~d}$ ). These intensity distributions are also clearly verified from the transverse line scans through the focus (see Figure 2e). It is of great interest to find from Figure $2 \mathrm{f}$ that the phase of the $z$-polarized component presents a helical structure, which indicates that through strongly focused optical system, the targeted light beam has become an optical vortex even though the incident light beam does not possess a vortex. This phenomenon occurs because, through a highly focused optical system, partial SAM of the incident circularly polarized light field could be transferred to OAM, which leads to a helical phase of the longitudinal component of the focal electric field.
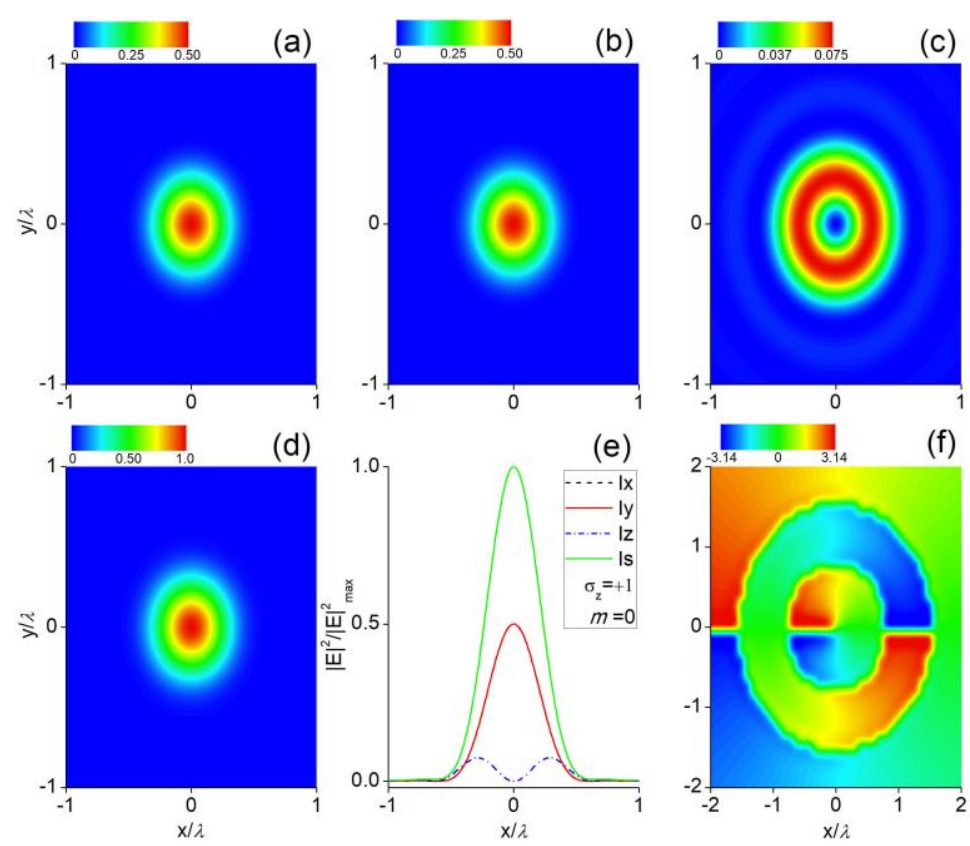

Figure 2. Calculated 2-D distributions $|E|^{2} /|E|^{2}$ max of a strongly focused RHC polarized $\left(\sigma_{z}=+1\right)$ optical vortex with charge $m=0$, i.e., Gaussian beam, without a FCL: (a) $x$-polarized component, (b) $y$-polarized component, (c) z-polarized component, (d) total field, (e) line scan of focus, and (f) the helical phase structure of the $z$-polarized component of the focal electric field.

Figure 3 illustrates the tight focusing of a RHC polarized Gaussian beam $\left(\sigma_{z}=+1, \mathrm{~m}=0\right)$ with a FCL of stage $S=3$. Comparing Figure $3 \mathrm{a}, \mathrm{b}$ with Figure $2 \mathrm{a}, \mathrm{b}$, one can obviously note that the transverse components have the same intensity distributions in cases with or without a FCL. Moreover, only a slight difference can be found in the magnitude of the longitudinal component $\left|E_{z}\right|^{2}$ between Figures $2 c$ and $3 c$. Thus, one may have an impression that the modulation effect of FCL on a tightly focused Gaussian beam is small and mainly reflected on the longitudinal component. However, the 
following simulations show that a FCL of different stage $S$ could significantly modulate the tight focusing intensity of circularly polarized optical vortex.
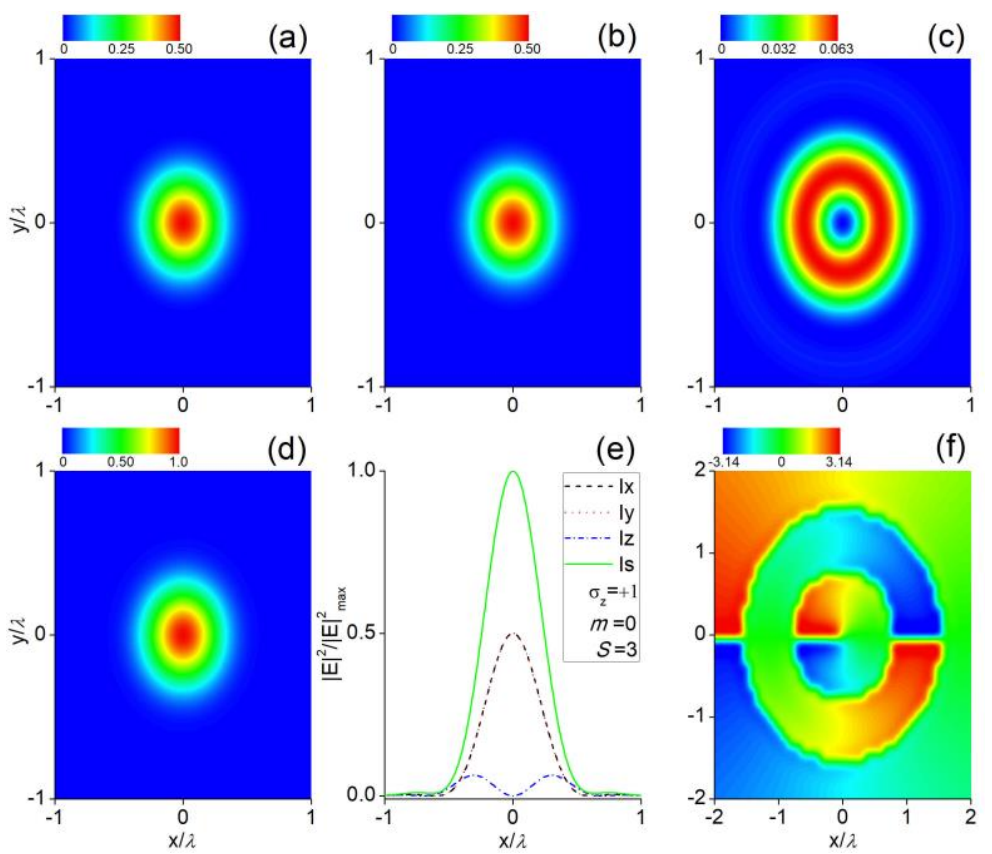

Figure 3. Calculated 2-D distributions of $|\mathrm{E}|^{2} /|\mathrm{E}|^{2}$ max for a strongly focused RHC polarized $\left(\sigma_{\mathrm{z}}=+1\right)$ optical vortex with charge $m=0$, i.e., Gaussian beam, regulated by a FCL of $S=3$ : (a) $x$-polarized component, (b) y-polarized component, (c) z-polarized component, (d) total field, (e) line scan of focus, and (f) the helical phase structure of the longitudinal component of the focal electric field.

Figure 4 depicts the strongly focused RHC polarized optical vortex $\left(\sigma_{z}=+1, m=1\right)$ with a FCL of stage $S=3$. In this case, the $x$-polarized component, $y$-polarized component and $z$-polarized component all present donut-shaped intensity distributions. As a result, the total intensity demonstrates a dark-centered distribution. In addition, the magnitude of $x$-polarized component and $y$-polarized component are identical, and meanwhile the z-polarized component's magnitude becomes much larger when compared with Figure 3. This phenomena can be explained as follows: for the RHC polarized vortex $\left(\sigma_{\mathrm{Z}}=+1, m=1\right)$, in the focal plane, the double helical phase appears (where $\exp [(m+1) i \varphi]=\exp (2 i \varphi)$ in Equation (6)) when the directions of the helical phase and circular polarization are the same, and it can be verified from the phase helical phase distribution of $z$-polarized electric field in Figure 4f. In addition, the dark hollow radius of the longitudinal polarized component depends on the topological charge, therefore, the dark area in Figure $4 \mathrm{c}$ becomes much wider than that in Figure 3c.

Figure 5 describes the distributions of strongly focused LHC polarized optical vortex $\left(\sigma_{z}=-1\right.$, $m=+1$ ) with a FCL of stage $S=3$. In this case, the direction of the helical phase is opposite to the direction of circular polarization $(\exp [(m-1) i \varphi]=1)$, which results in the $z$-polarized component electric field no longer possessing a vortex, and thereby no helical phase exists in the $z$-polarized component (see Figure 5f). Although both $x$-polarized and $y$-polarized component intensities maintain a donut-shaped configuration (see Figure $5 \mathrm{a}, \mathrm{b}$ ), the $z$-polarized component generates a peak-centered distribution (Figure 5c). As a result, the total field produces a much wider bright area in the intensity distribution (see Figure 5d). 

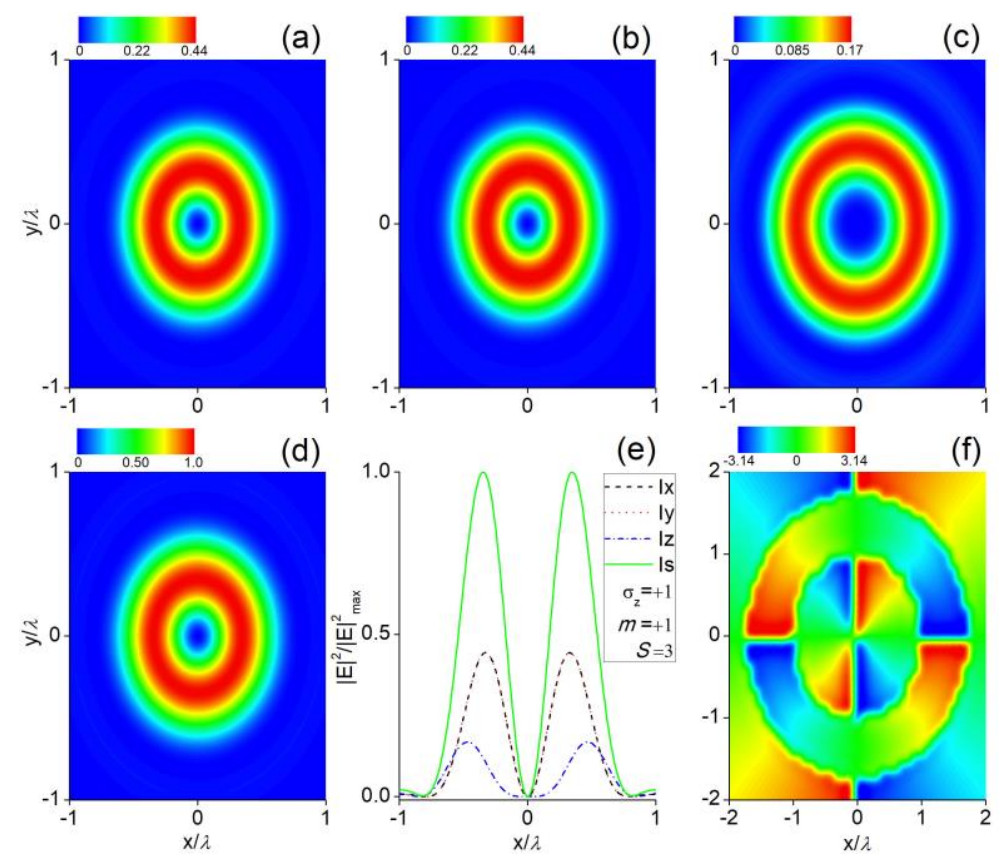

Figure 4. Calculated 2D distributions of $|\mathrm{E}|^{2} /|\mathrm{E}|^{2}$ max for a strongly focused RHC polarized $\left(\sigma_{\mathrm{z}}=+1\right)$ optical vortex with charge $m=+1$, regulated by a FCL of $S=3$ : (a) $x$-polarized component, (b) $y$-polarized component, (c) z-polarized component, (d) total field, (e) line scan of focus, and (f) the helical phase structure of the longitudinal component of the focal electric field.
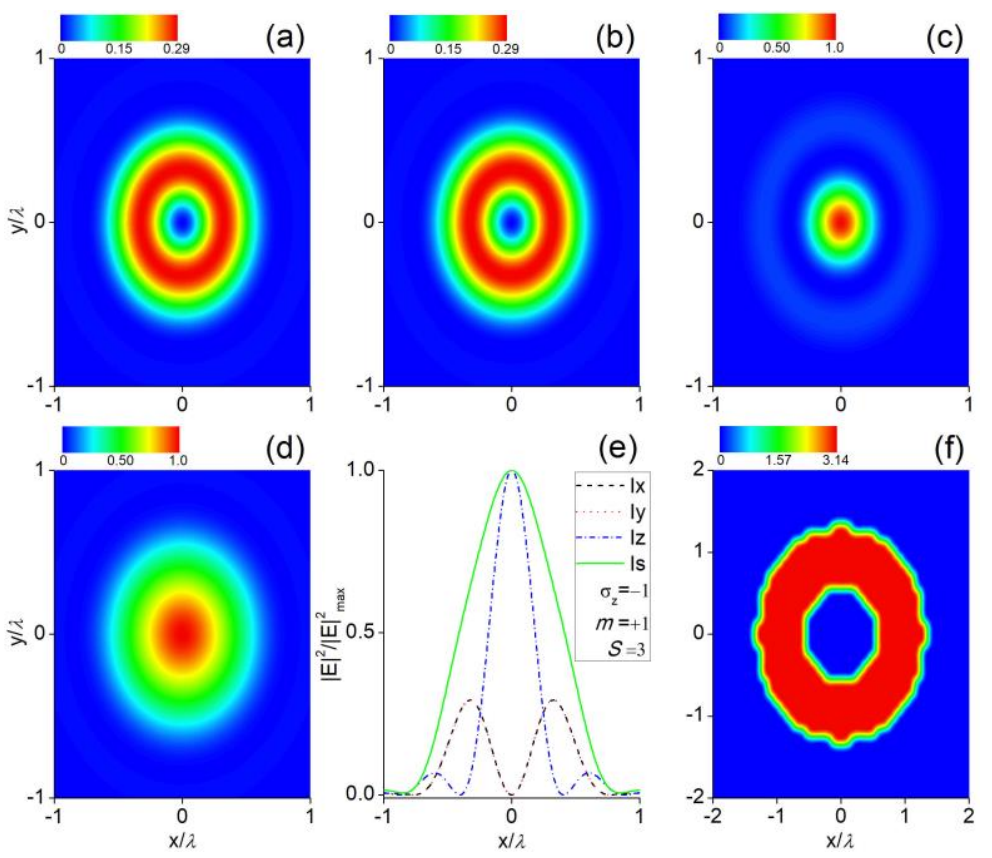

Figure 5. Calculated $2 \mathrm{D}$ distributions $|\mathrm{E}|^{2} /|\mathrm{E}|^{2}$ max for a strongly focused LHC polarized $\left(\sigma_{\mathrm{z}}=-1\right)$ optical vortex with charge $m=+1$, regulated by a FCL of $S=3$ : (a) $x$-polarized component, (b) $y$-polarized component, (c) z-polarized component, (d) total field, (e) line scan of focus, and (f) the helical phase structure of the longitudinal component of the focal electric field.

Figure 6 shows the evolution of the $z$-polarized component intensity Iz (upper row) and the total intensity Is (lower row) (2D left column, and 1D right column, the Figures 7-10 are the same) of strongly focused RHC Gaussian beams without a FCL. As can be noted from Figure 6a,b, for the strongly focused Gaussian beam $\left(\sigma_{z}=+1, m=0\right)$, the $z$-polarized component produces an on-axis null along the 
$z$-axis, whereas the total intensity reaches its maximum in the focal point (see Figure 1c,d). Additionally, both the $z$-polarized component intensity and the total intensity have symmetric distributions about the focal point.

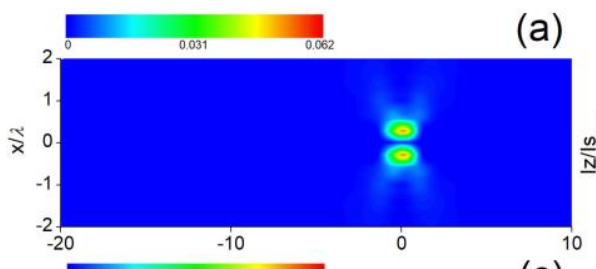

(a)

(b)
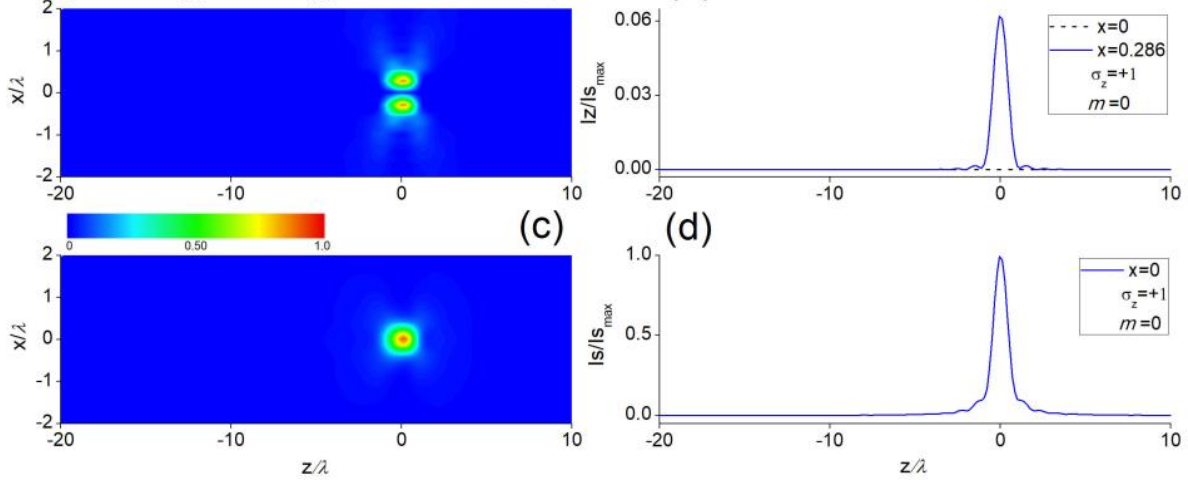

Figure 6. Evolution of the z-polarized component intensity Iz/IS $\max (\mathbf{a}, \mathbf{b})$ and the total intensity Is/Is $\max$ $(\mathbf{c}, \mathbf{d})(2 \mathrm{D}(\mathbf{a}, \mathbf{c})$, and $1 \mathrm{D}(\mathbf{b}, \mathbf{d}))$ of strongly focused RHC polarized $\left(\sigma_{\mathrm{Z}}=+1\right)$ optical vortex $\mathrm{LG}_{0}^{m}(m=0)$, i.e., Gaussian beam, without a FCL.

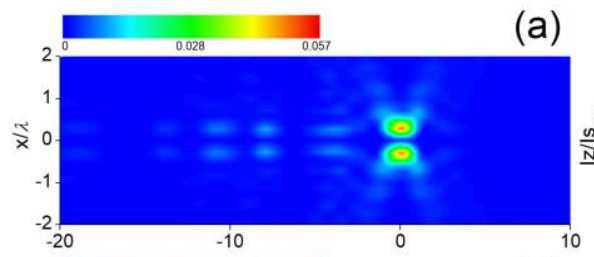

(a)
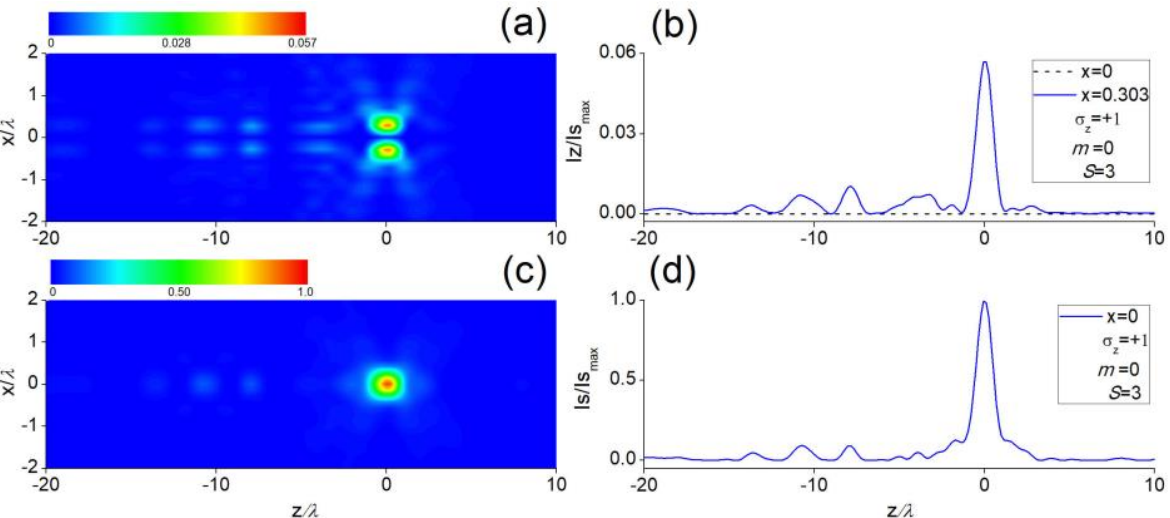

Figure 7. Evolution of the z-polarized component intensity Iz/Is $\mathrm{s}_{\max }(\mathbf{a}, \mathbf{b})$ and total intensity $\mathrm{Is} / \mathrm{Is} \max$ $(\mathbf{c}, \mathbf{d})(2 \mathrm{D}(\mathbf{a}, \mathbf{c})$, and $1 \mathrm{D} \mathbf{b}, \mathbf{d})$ of strongly focused RHC polarized $\left(\sigma_{\mathbf{Z}}=+1\right)$ optical vortex $\mathrm{LG}_{0}^{m}(m=0)$, i.e., Gaussian beam, regulated by a FCL of stage $S=3$.
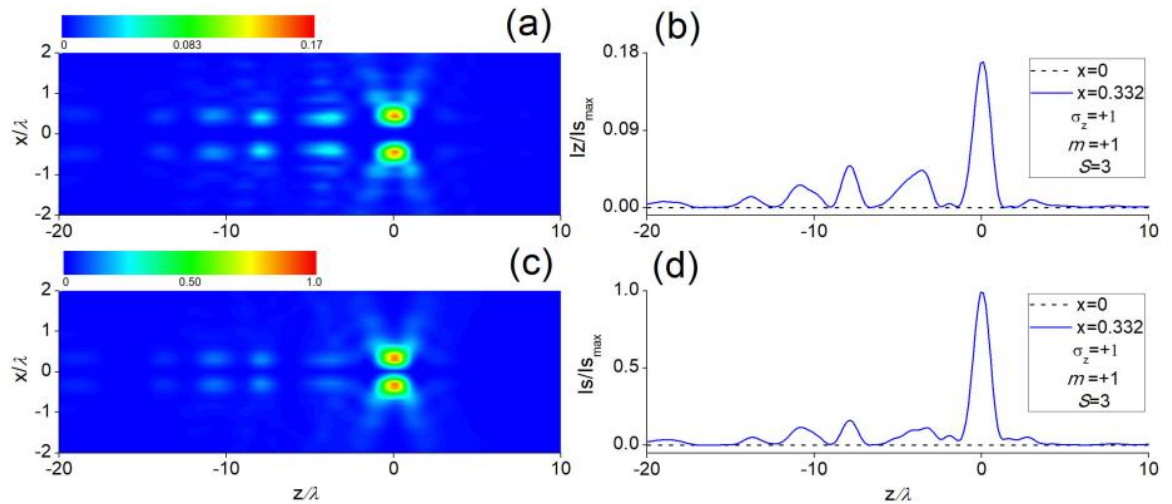

Figure 8. Evolution of the $z$-polarized component intensity $\mathrm{Iz} / \mathrm{Is}_{\max }(\mathbf{a}, \mathbf{b})$ and total intensity $\mathrm{Is} / \mathrm{Is}_{\max }$ $(\mathbf{c}, \mathbf{d})(2 \mathrm{D}(\mathbf{a}, \mathbf{c})$, and $1 \mathrm{D}(\mathbf{b}, \mathbf{d}))$ of strongly focused RHC polarized $\left(\sigma_{\mathrm{z}}=+1\right)$ optical vortex $\mathrm{LG}_{0}^{m}(m=+1)$ and $\sigma_{\mathrm{z}}=+1$ regulated by a FCL of stage $S=3$. 

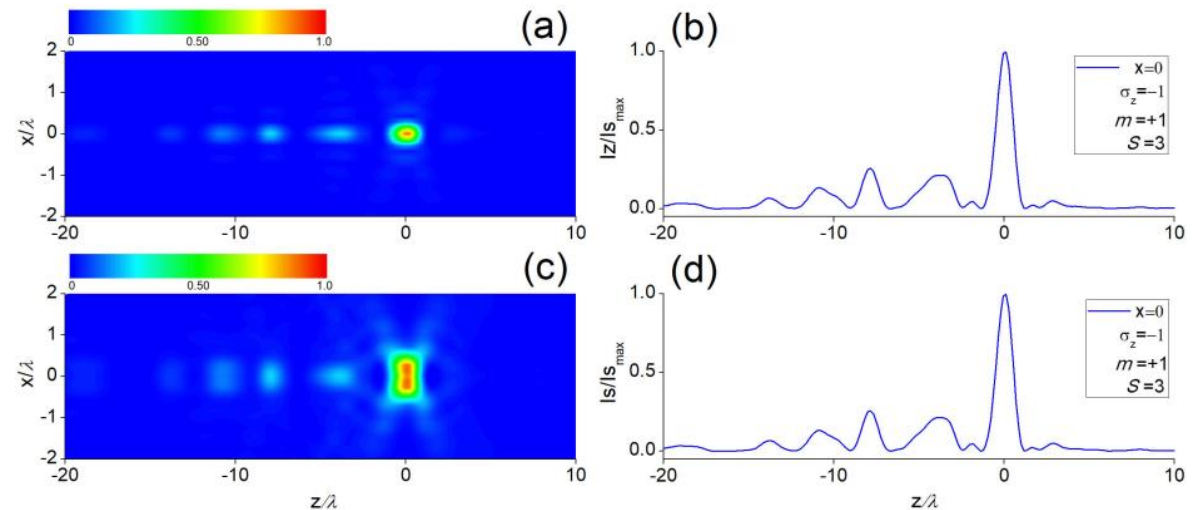

Figure 9. Evolution of the $z$-polarized component intensity Iz/Is $\max (\mathbf{a}, \mathbf{b})$ and the total intensity Is/Is $\max$ $(\mathbf{c}, \mathbf{d})(2 \mathrm{D}(\mathbf{a}, \mathbf{c})$, and $1 \mathrm{D}(\mathbf{b}, \mathbf{d}))$ of strongly focused LHC polarized $\left(\sigma_{z}=-1\right)$ optical vortex $\mathrm{LG}_{0}^{m}(m=+1)$ regulated by a FCL of stage $S=3$.
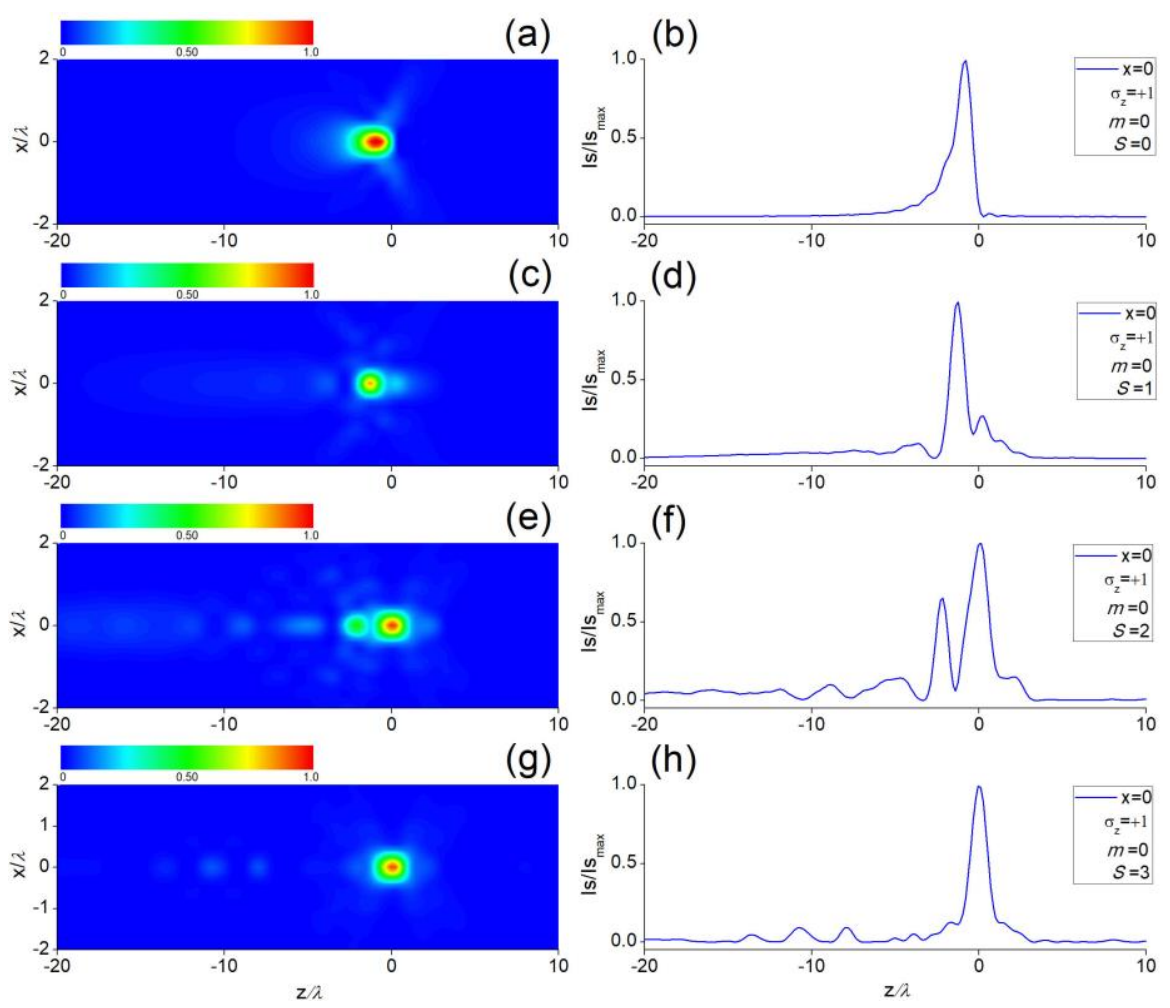

Figure 10. Evolution of the total intensity Is/Is $\max (2 \mathrm{D}(\mathbf{a}, \mathbf{c}, \mathbf{e}, \mathbf{g})$, and $1 \mathrm{D}(\mathbf{b}, \mathbf{d}, \mathbf{f}, \mathbf{h}))$ of strongly focused RHC $\left(\sigma_{\mathrm{Z}}=+1\right)$ polarized optical vortex $\operatorname{LG}_{0}^{m}(m=0)$ regulated by a FCL of stage $S=0,1,2$, and 3 , respectively.

Figure 7 depicts evolution of the $z$-polarized component intensity Iz (upper row) and the total intensity Is (lower row) of strongly focused RHC Gaussian beams $\left(\sigma_{z}=+1, m=0\right)$ regulated by a FCL of stage $S=3$. It is clearly seen from Figure 7 that, in the presence of a FCL, both the $z$-polarized component and the total intensity exhibit a single major focus and several subsidiary focal points surrounding it, which leads to neither the z-polarized component nor the total intensity maintaining symmetry about the focal point.

When the incident optical field is a RHC polarized optical vortex $\mathrm{LG}_{0}^{m}$ with $\sigma_{\mathrm{z}}=+1$ and $m=+1$, the high NA optical system is regulated by a FCL of stage $S=3$, as shown in Figure 8. Because of FCL's self-similarity diffractive properties, in the focal region, several subsidiary focal points are distributed 
surrounding the major focus along the $z$-axis; additionally, both the $z$-polarized component and the total intensity maintain on-axis null along the $z$-axis (see Figure 8).

If the incident beam is a LHC polarized optical vortex $\mathrm{LG}_{0}^{m}$ with $\sigma_{\mathrm{z}}=-1$ and $m=+1$, the high NA optical system is regulated by a FCL of stage $S=3$, as shown in Figure 9. In this case, the helical phase and the circular polarization have opposite directions, therefore, the photon has no OAM, which causes both the $z$-polarized component and the total intensity distributions to have a principle maximum in the focal point and several sub maximums located nearby along the $z$-axis.

Figure 10 studies the influence of FCL's stage $S$ on the evolution of total intensity of strongly focused RHC polarized optical vortex $\mathrm{LG}_{0}^{m}$ with $\sigma_{\mathrm{z}}=+1$ and $m=0$, where FCL's stage $S$ is chosen as $0,1,2$, and 3, respectively. As can be found from Figure 10, in the presence of a FCL the total intensity distributions will not maintain symmetry about the focal plane any more. Furthermore, more subsidiary focal points emerge when increasing FCL's stage $S$.

\section{Conclusions}

In this work, we introduced a recently-proposed pure-phase optical element, a fractal conical lens (FCL), to a high NA optical system. Following the generalized vector Debye integral, tight focusing characteristics of a circularly polarized optical vortex through a high NA optical system in cases with and without a FCL were investigated comparatively. Moreover, the conversion between the SAM and OAM of the focused vortex beams in the focal vicinity was also analyzed. In addition, dependence of several factors, such as incident polarization $\sigma_{\mathrm{z}}$, optical vortex's topological charge $m$, FCL's stage $S$, and objective lens' NA, on the circularly polarized optical vortex's focusing properties and the conversion between the SAM and OAM in the focal vicinity were investigated in detail. It was found that these factors all played significant roles in determining the targeted optical vortex's focusing intensity distribution and the conversion between SAM and OAM. These interesting findings might be advantageous when using a FCL to shape vortex beams or utilizing circularly polarized vortex beams to exploit new-type optical tweezers.

Author Contributions: Data curation, Z.L.; writing original draft, K.H., A.Y., and X.W.; supervision, P.H.J.; project administration, Z.L. All authors have read and agreed to the published version of the manuscript.

Funding: This work was supported by the National Natural Science Foundation of China $(11764015,11864013)$, and the Scientific Project of Jiangxi Education Department of China (GJJ170361).

Conflicts of Interest: The authors declare no conflict of interest.

\section{References}

1. Richards, B.; Wolf, E. Electromagnetic diffraction in optical systems, 2. structure of the image field in an aplanatic system. Proc. R. Soc. Lond. A 1959, 253, 358-379.

2. Dorn, R.; Quabis, S.; Leuchs, G. Sharper focus for a radially polarized light beam. Phys. Rev. Lett. 2003, 91, 233901. [CrossRef] [PubMed]

3. Youngworth, K.S.; Brown, T.G. Focusing of high numerical aperture cylindrical vector beams. Opt. Express 2000, 7, 77-87. [CrossRef] [PubMed]

4. Zhan, Q.; Leger, J.R. Focus shaping using cylindrical vector beams. Opt. Express 2002, 10, 324-331. [CrossRef] [PubMed]

5. Zhan, Q. Properties of circularly polarized vortex beams. Opt. Lett. 2006, 31, 867-869. [CrossRef]

6. Yuan, G.H.; Wei, S.B.; Yuan, X.C. Nondiffracting transversally polarized beam. Opt. Lett. 2011, 36, 3479-3481. [CrossRef]

7. Tian, B.; Pu, J. Tight focusing of a double-ring-shaped, azimuthally polarized beam. Opt. Lett. 2011, 36, 2014-2016. [CrossRef]

8. $\mathrm{Hu}, \mathrm{K}$; Chen, Z.; Pu, J. Generation of super-length optical needle by focusing hybridly polarized vector beams through a dielectric interface. Opt. Lett. 2012, 37, 3303-3305. [CrossRef]

9. Nieminen, T.A.; Heckenberg, N.R.; Rubinsztein-Dunlop, H. Forces in optical tweezers with radially and azimuthally polarized trapping beams. Opt. Lett. 2008, 33, 122-124. [CrossRef] 
10. Gilboa, T.; Zrehen, A.; Girsault, A.; Meller, A. Optically-Monitored Nanopore Fabrication Using a Focused aser Beam. Sci. Rep. 2018, 8, 9765. [CrossRef]

11. Li, M.; Yan, S.; Zhang, Y. Optical sorting of small chiral particles by tightly focused vector beams. Phys. Rev. A 2019, 99, 033825. [CrossRef]

12. Cai, P.; Zha, J.; Xie, Y. Rydberg-atom acceleration by tightly focused intense laser pulses. Phys. Rev. A 2019, 99, 053401. [CrossRef]

13. Sheppard, C.J.R. High-aperture beams. J. Opt. Soc. Am. A 2001, 18, 1579-1587. [CrossRef] [PubMed]

14. Dorn, R.; Quabis, S.; Leuchs, G. The focus of light-linear polarization breaks the rotational symmetry of the focal spot. J. Mod. Opt. 2003, 50, 1917-1926.

15. Quabis, S.; Dorn, R.; Eberler, M.; Glockl, O.; Leuchs, G. Focusing light to a tighter spot. Opt. Commun. 2000, 179, 1-7. [CrossRef]

16. Scully, M.O.; Zubairy, M.S. Simple laser accelerator: Optics and particle dynamics. Phys. Rev. A 1991, 44, 2656-2663. [CrossRef] [PubMed]

17. Zurita-Sa'nchez, J.R.; Novotny, L. Multipolar interband absorption in a semiconductor quantum dot. II. Magnetic dipole enhancement. J. Opt. Soc. Am. B 2002, 19, 2722-2726.

18. Poynting, J.H. The wave motion of a revolving shaft, and a suggestion as to the angular momentum in a beam of circularly polarized light. Proc. R. Soc. Lond. Ser. A 1909, 82, 560-567. [CrossRef]

19. Allen, L.; Beijersbergen, M.W.; Spreeuw, R.J.C.; Woerdman, J.P. Orbital angular-momentum of light and the transformation of Laguerre-Gaussian laser modes. Phys. Rev. A 1992, 45, 8185-8189. [CrossRef]

20. Marrucci, L.; Manzo, C.; Paparo, D. Optical spin-to-orbital momentum conversion in inhomogeneous anisotropic media. Phys. Rev. Lett. 2006, 96, 163905. [CrossRef]

21. Zhao, Y.; Edgar, J.S.; Jeffries, G.D.M.; McGloin, D.; Chiu, D.T. Spin-to-orbital angular momentum conversion in a strongly focused optical beam. Phys. Rev. Lett. 2007, 99, 073901. [CrossRef] [PubMed]

22. Chalice, D.R. A characterization of the cantor function. Am. Math. Mon. 1991, 98, 255-258. [CrossRef]

23. Monsoriu, J.A.; Furlan, W.D.; Andrés, P.; Lancis, J. Fractal conical lenses. Opt. Express 2006, 14, 9077-9082. [CrossRef] [PubMed]

24. Cheng, S.; Zhang, X.; Ma, W.; Tao, S. Fractal zone plate beam based optical tweezers. Sci. Rep. 2016, 6, 34492. [CrossRef]

25. Gu, M. Advanced Optical Imaging Theory; Springer-Verlag: Berlin/Heidelberg, Germany, 1999. 